\title{
Evaluation of diffuse fraction and diffusion coefficient using statistical analysis
}

\author{
Hicham Salhi ${ }^{1} \cdot$ Lazhar Belkhiri $^{1} \cdot$ Ammar Tiri $^{1}$
}

Received: 10 January 2020 / Accepted: 28 April 2020 / Published online: 12 May 2020

(c) The Author(s) 2020

\begin{abstract}
In this study, eighty models are proposed in order to estimate the diffuse fraction and diffuse coefficient. For the proposed models, sunshine ratio and clearness index are considered as predictors. Monthly average global and diffuse solar radiation together with sunshine duration data of Tamanrasset station form 1995 to 2017 were analyzed. The different proposed models are compared and statistically analyzed to assess the performance of the best fitted model. Nine statistical indicators and Global Performance Indicator are computed to evaluate different proposed models. It is concluded that the cubic model with sunshine ratio and clearness index is selected as the best accurate model to estimate diffuse solar radiation on a horizontal surface in the study area.
\end{abstract}

Keywords Statistical analysis - Diffuse fraction $\cdot$ Diffusion coefficient $\cdot$ Sunshine ratio $\cdot$ Clearness index $\cdot$ Tamanrasset station

\section{List of symbols}

$H_{\mathrm{d}}$ Diffuse solar radiation on a horizontal surface (MJ/ $\mathrm{m}^{2}$ day)

$H$ Global solar radiation on a horizontal surface (MJ/ $\mathrm{m}^{2}$ day)

$H_{0} \quad$ Extraterrestrial radiation

$k_{\mathrm{d}} \quad$ Diffuse fraction (or cloudiness index)

$K_{\mathrm{D}} \quad$ Diffusion coefficient

$K_{t} \quad$ Clearness index

$S_{t} \quad$ Sunshine ratio

$S_{o} \quad$ Maximum possible sunshine duration (h)

$S \quad$ Sunshine duration (h)

Hicham Salhi

salhiheat@gmail.com

Lazhar Belkhiri

belkhiri_laz@yahoo.fr; belkhiri.la@gmail.com

Ammar Tiri

Tiri_ammar@yahoo.fr

1 Laboratory of Applied Research in Hydraulics, University of Mustapha Ben Boulaid-Batna 2, Batna, Algeria

\section{Introduction}

Solar energy is a renewable energy resource in nature, and it plays a major factor in between other alternative energy source. For any study of solar energy, the information of solar radiation at a given geographical location is very important (Bakirci 2009, 2015). There are many important radiation parameters such as global, diffuse and direct radiation used in solar energy techniques (Hussain et al. 1999). Solar energy in Algeria is available in abundant amounts across the year; the average duration of sunshine value is $3000 \mathrm{~h} /$ year. Also, the average energy is $1700 \mathrm{KW} \mathrm{h} / \mathrm{m}^{2} /$ year in the North and $2650 \mathrm{KW} \mathrm{h} / \mathrm{m}^{2} /$ year in the South (BoudgheneStambouli 2011; Bouchouicha et al. 2015). There are many studies carried out in the world for estimating diffuse solar radiation using the available data which include hours of solar radiation (Sabbagh et al. 1977; Iqbal 1979; Erbs et al. 1982; De Miguel et al. 2001; Paliatsos et al. 2003; Li et al. 2012).

The recent studies have used empirical models based on mathematical function to estimate the diffuse radiation using clearness index and sunshine ratio, thus playing an essential role in the absence of required technological installations. Many authors have used linear or nonlinear regression models and polynomial models to correlate diffusion coefficient or diffuse fraction with sunshine ratio and/or clearness index (Orgill and Hollands 1977; Spencer 
1982; Reindl et al. 1990; Lam and Li 1996; Hua et al. 2002; Soares et al. 2004). Kuo et al. (2014) studied the data for global and diffuse radiation in Tainan, Taiwan, for two years; the proposed models are compared with the fourteen models available in the literature; it is concluded that the proposed piece-wise linear models perform well in predicting the diffuse fraction. Liu et al. (2017) developed four models using global solar radiation and sunshine duration data in China; the analysis of statistical indexes demonstrated that cubic models presented the best performance in radiation zone. For Algeria, Mecibah et al. (2014) proposed quadric and cubic models based on the sunshine-based models. Also, Bailek et al. (2017) reviewed and compared thirty-five proposed correlations to measured irradiation of Algerian Big South (Adrar region); it is concluded that the second-order polynomial model of diffuse fraction is able to estimate the monthly average daily diffuse irradiation on a horizontal surface.

The main objective of this study is to develop and compare different proposed empirical models for estimation of horizontal monthly mean diffuse solar radiation based on clearness index and sunshine ratio.

\section{Methodology}

\section{Solar radiation data}

Data of horizontal global solar radiation, diffuse solar radiation and sunshine period of Tamanrasset station were taken from National Meteorological Office of Algeria from 1995 to 2017. The geographical information of the station is given in Table 1. Tamanrasset is located in the southeastern region of Algeria (Fig. 1). Tamanrasset has a hot desert climate (Köppen climate classification BWh), with very hot summers and mild winters. There is very little rain throughout the year, although occasional rain does fall in late summer from the northern extension of the Intertropical Convergence Zone.

Table 1 Geographic and data records period of Tamanrasset

\begin{tabular}{ll}
\hline Latitude $\left({ }^{\circ} \mathrm{N}\right)$ & 22.78 \\
Longitude $\left({ }^{\circ} \mathrm{E}\right)$ & 5.51 \\
Elevation $(\mathrm{m})$ & 1378 \\
Data series period & $1995-2017$ \\
Mean $\mathrm{GH}\left(\mathrm{MJ} / \mathrm{m}^{2}\right.$ day) & 7.26 \\
Mean $S(\mathrm{~h})$ & 9.20 \\
Mean $T^{\circ}\left({ }^{\circ} \mathrm{C}\right)$ & 22.71 \\
Mean $\mathrm{RH}(\%)$ & 28.6 \\
\hline
\end{tabular}

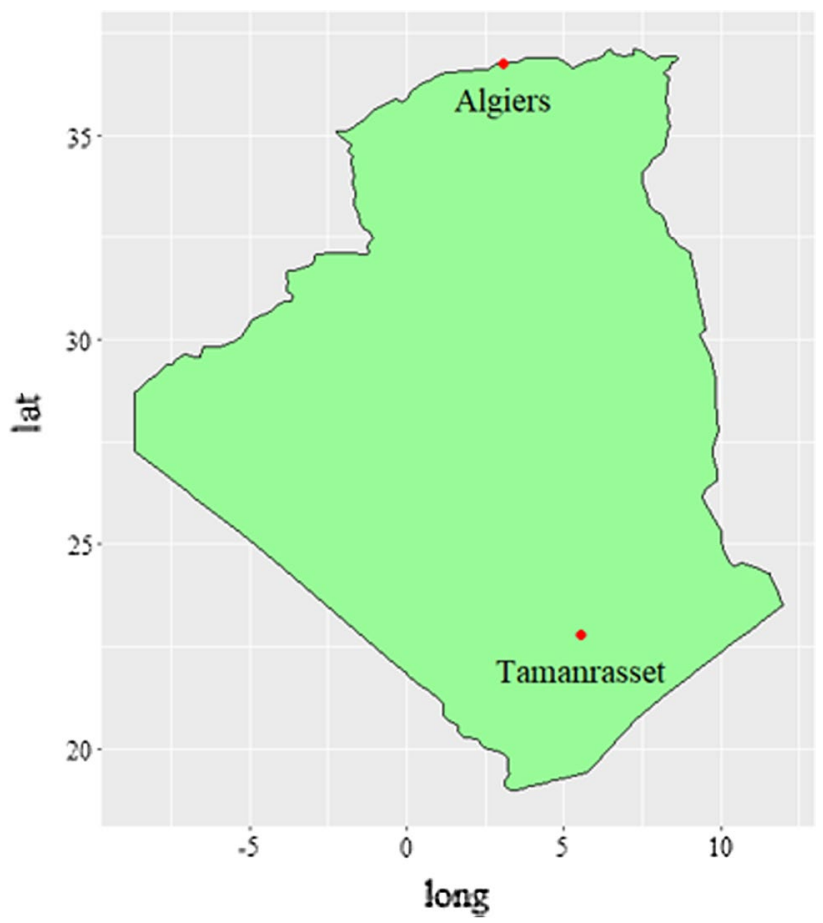

Fig. 1 Location of Tamanrasset station

\section{Proposed of models}

In the current study, the regression analysis is used for the proposed models, where the predictand is the diffuse fraction $\left(k_{\mathrm{d}}\right)$ or diffuse coefficient $\left(K_{\mathrm{D}}\right)$ and the predictors are sunshine ratio $\left(S_{\mathrm{t}}\right)$ and clearness index $\left(K_{\mathrm{t}}\right)$. Thus, three types of forty models can be defined for e diffuse fraction and diffusion coefficient. The three respective types can be written as:

$\left(k_{\mathrm{d}}=\frac{H_{\mathrm{d}}}{H}, K_{\mathrm{D}}=\frac{H_{\mathrm{d}}}{H_{0}}\right) \approx f\left(S_{t}\right)$

$\left(k_{\mathrm{d}}=\frac{H_{\mathrm{d}}}{H}, K_{\mathrm{D}}=\frac{H_{\mathrm{d}}}{H_{0}}\right) \approx f\left(K_{t}\right)$

$\left(k_{\mathrm{d}}=\frac{H_{\mathrm{d}}}{H}, K_{\mathrm{D}}=\frac{H_{\mathrm{d}}}{H_{0}}\right) \approx f\left(S_{t}, K_{t}\right)$

where $H_{0}, H$, and $H_{\mathrm{d}}$ are the monthly mean daily extraterrestrial solar radiation, global solar radiation and diffuse solar radiation on a horizontal surface, respectively. Mathematically, sunshine ratio and clearness index are defined as

$K_{t}=\frac{H}{H_{0}}$ 
$S_{t}=\frac{S}{S_{0}}$

where $S$ and $S_{\mathrm{o}}$ are the sunshine duration and maximum possible sunshine durations, respectively.

The monthly average daily extraterrestrial solar radiation on a horizontal surface is calculated from the following equation Klein (1977):

$$
\begin{aligned}
H_{0}= & \frac{24}{\pi} H_{\mathrm{sc}}\left(1+0.033 \cos \left(\frac{360}{365} n\right)\right) \\
& \left(\cos \varphi \cos \delta \sin \omega_{\mathrm{s}}+\frac{\pi}{180} \omega_{\mathrm{s}} \sin \varphi \sin \delta\right)
\end{aligned}
$$

where $H_{\text {sc }}$ is the solar constant, $n$ is the Julian day of the year, $\varphi$ is the location latitude, and $\delta$ is declination angle, $\omega_{\mathrm{s}}$ is the sunset hour angle. $\delta$ and $\omega_{\mathrm{s}}$ are mathematically defined as:

$\delta=23.45 \sin \left[360 \frac{(n+284)}{365}\right]$

$\omega_{\mathrm{s}}=\cos ^{-1}[-\tan \varphi \tan \delta]$

The maximum possible sunshine duration $\left(S_{0}\right)$ is calculated as:

$S_{0} \frac{2}{15} \omega_{\mathrm{s}}$

The forty proposed models for each diffuse fraction and diffusion coefficient are presented in Table 2.

\section{Statistical evaluation}

In this study, nine statistical indicators were used to evaluate different proposed models such as mean bias error (MBE), mean absolute error (MAE), mean absolute relative error (MARE), mean absolute percentage error (MAPE), root mean squared error (RMSE), root mean squared relative error (RMSRE), relative root mean squared error (RRMSE), correlation coefficient $\left(R^{2}\right)$ and $t$-statistics ( $t$-stat). Mathematical expressions for these indicators are defined as:

$$
\begin{aligned}
& \text { MBE }=\frac{1}{n} \sum_{i=1}^{n}\left(H_{i, o}-H_{i, m}\right) \\
& \text { MAE }=\frac{1}{n} \sum_{i=1}^{n}\left|H_{i, m}-H_{i, o}\right| \\
& \text { MARE }=\frac{1}{n} \sum_{i=1}^{n}\left|\frac{H_{i, m}-H_{i, o}}{H_{i, m}}\right|
\end{aligned}
$$

MAPE $=\frac{1}{n} \sum_{i=1}^{n}\left|\frac{H_{i, m}-H_{i, o}}{H_{i, o}}\right| * 100$

$\mathrm{RMSE}=\sqrt{\frac{1}{n} \sum_{i=1}^{n}\left(H_{i, m}-H_{i, o}\right)^{2}}$

$\operatorname{RMSRE}=\sqrt{\frac{1}{n} \sum_{i=1}^{n}\left(\frac{H_{i, m}-H_{i, o}}{H_{i, m}}\right)^{2}}$

$\operatorname{RRMSE}=\sqrt{\frac{\frac{1}{n} \sum_{i=1}^{n}\left(H_{i, m}-H_{i, o}\right)^{2}}{\frac{1}{n} \sum_{i=1}^{n} H_{i, m}}} * 100$

$R^{2}=1-\frac{\sum_{i=1}^{n}\left(H_{i, m}-H_{i, o}\right)^{2}}{\sum_{i=1}^{n}\left(H_{i, m}-\overline{H_{m}}\right)}$

$t-$ stat $=\sqrt{\frac{(n-1) \mathrm{MBE}^{2}}{\mathrm{RMSE}^{2}-\mathrm{MBE}^{2}}}$

where $n$ is the number of the solar irradiation data, $H_{i, m}$ is the $i$ th estimated values, $H_{i, o}$ is the $i$ th observed values and $\overline{H_{m}}$ is the estimated mean value.

\section{Results and discussion}

The statistical descriptive of diffuse solar radiation includes minimum, maximum, mean, standard deviation and coefficient of variance is shown in Table 3. The mean value of global and diffuse solar radiation is 2320 and $704.2 \mathrm{MJ} /$ $\mathrm{m}^{2}$ day with a standard deviation of 394.4 and $282.35 \mathrm{MJ} /$ $\mathrm{m}^{2}$ day, respectively. The coefficient of variance of $H$ and $H_{\mathrm{d}}$ is 17 and $40 \%$, respectively. The diffuse fraction and diffusion coefficient values ranged from 0.105 to 0.577 and 0.030 to 0.121 with a mean of 0.298 and 0.074 , respectively. High values of $k_{\mathrm{d}}$ and $K_{\mathrm{D}}$ are observed in the months of April, May, June, July, August and September (Fig. 2).

The values of sunshine ratio and clearness index varied from 0.205 to 0.298 and 7.105 to 16.401 with a mean of 0.251 and 12.826 , respectively, where high values for both predictors $\left(S_{t}\right.$ and $\left.K_{t}\right)$ are observed between the months of January to December (Fig. 2). From the Fig. 3, it was observed that there is a significant negative correlation between $k_{\mathrm{d}}-S_{t}(-0.859), k_{\mathrm{d}}-K_{t}(-0.722), K_{\mathrm{D}}-S_{t}(-0.825)$, and $K_{\mathrm{D}}-K_{t}(-0.577)$.

The statistical indicators of the different models are given in Table 4. For higher modeling accuracy MBE, 
Table 2 (a) Various forms of correlations for diffuse fraction and (b) various forms of correlations for diffusion coefficient

\begin{tabular}{|c|c|c|}
\hline & Models & Forms of the models \\
\hline \multicolumn{3}{|l|}{ (a) } \\
\hline \multirow[t]{5}{*}{$k_{\mathrm{d}} \approx f\left(S_{t}\right)$} & M1 & $K_{\mathrm{d}}=0.8671-0.044 S_{t}$ \\
\hline & M2 & $K_{\mathrm{d}}=0.6498-0.0084 S_{t}-0.0014 S_{t}^{2}$ \\
\hline & M3 & $K_{\mathrm{d}}=0.27947+0.0884 S_{t}-0.0096 S_{t}^{2}+0.00022 S_{t}^{3}$ \\
\hline & M4 & $K_{\mathrm{d}}=1.6363-0.527 \log \left(S_{t}\right)$ \\
\hline & M5 & $K_{\mathrm{d}}=0.338-3.165 e-08\left(S_{t}\right)$ \\
\hline \multirow[t]{5}{*}{$k_{\mathrm{d}} \approx f\left(K_{t}\right)$} & M6 & $K_{\mathrm{d}}=1.244-3.7667 K_{t}$ \\
\hline & M7 & $K_{\mathrm{d}}=2.7023-15.5047 K_{t}+23.4966 K_{t}^{2}$ \\
\hline & M8 & $K_{\mathrm{d}}=-2.149+43.268 K_{t}-212.6799 K_{t}^{2}+314.852 K_{t}^{3}$ \\
\hline & M9 & $K_{\mathrm{d}}=-1.0036-0.9405 \log \left(K_{t}\right)$ \\
\hline & M10 & $K_{\mathrm{d}}=4.0634-2.9283 \exp \left(K_{t}\right)$ \\
\hline \multirow[t]{29}{*}{$k_{\mathrm{d}} \approx f\left(S_{t}, K_{t}\right)$} & M11 & $K_{\mathrm{d}}=1.0773-1.284 K_{t}-0.0356 S_{t}$ \\
\hline & M12 & $K_{\mathrm{d}}=0.862-1.2834-0.0014 S_{t}^{2}$ \\
\hline & M13 & $K_{\mathrm{d}}=0.8038-1.3563 K_{t}-0.000073 S_{t}^{3}$ \\
\hline & M14 & $K_{\mathrm{d}}=1.5634-5.21 K_{t}+7.8084 K_{t}^{2}-0.03525 S_{t}$ \\
\hline & M15 & $K_{\mathrm{d}}=1.6769-7.8387 K_{t}+13.0676 K_{t}^{2}-0.0014 S_{t}^{2}$ \\
\hline & M16 & $K_{\mathrm{d}}=1.9146-10.289 K_{t}+17.8432 K_{t}^{2}-7.3165 e-05 S_{t}^{3}$ \\
\hline & M17 & $K_{\mathrm{d}}=1.732-8.9753 K_{t}+15.2995 K_{t}^{2}+0.0143 S_{t}-0.0019 S_{t}^{2}$ \\
\hline & M18 & $K_{\mathrm{d}}=1.3754-9.2057 K_{t}+15.7663 K_{t}^{2}+0.1147 S_{t}-0.0105 S_{t}^{2}+0.00023 S_{t}^{3}$ \\
\hline & M19 & $K_{\mathrm{d}}=1.7641-8.7455 K_{t}+14.8467 K_{t}^{2}-8.88 e-04 S_{t}^{2}-2.769 e-05 S_{t}^{3}$ \\
\hline & M20 & $K_{\mathrm{d}}=6.8129-68.8458 K_{t}+263.825 K_{t}^{2}-341.546 K_{t}^{3}-0.0356 S_{t}$ \\
\hline & M21 & $K_{\mathrm{d}}=10.898-120.453 K_{t}+462.173 K_{t}^{2}-594.048 K_{t}^{3}+241.606 S_{t}-2.412 e-03 S_{t}^{2}$ \\
\hline & M22 & $K_{\mathrm{d}}=10.548-114.886 K_{t}+440.302 K_{t}^{2}-565.56 K_{t}^{3}-0.00561 S_{t}-6.385 e-05 S_{t}^{3}$ \\
\hline & M23 & $K_{\mathrm{d}}=12.261-145.598 K_{t}+562.111 K_{t}^{2}-725.735 K_{t}^{3}+0.214 S_{t}-0.0184 S_{t}^{2}+4.42 e-04 S_{t}^{3}$ \\
\hline & M24 & $K_{\mathrm{d}}=10.465-114.178 K_{t}+437.801 K_{t}^{2}-562.654 K_{t}^{3}-5.79 e-04 S_{t}^{2}-4.54 e-05 S_{t}^{3}$ \\
\hline & M25 & $K_{\mathrm{d}}=9.3589-100.998 K_{t}+387.842 K_{t}^{2}-499.905 K_{t}^{3}-0.00145 S_{t}^{2}$ \\
\hline & M26 & $K_{\mathrm{d}}=11.138-122.15 K_{t}+467.77 K_{t}^{2}-600.016 K_{t}^{3}-7.517 e-05 S_{t}^{3}$ \\
\hline & M27 & $K_{\mathrm{d}}=1.3911-3.1948 K_{t}+10.027 K_{t}^{3}-0.0352 S_{t}$ \\
\hline & M28 & $K_{\mathrm{d}}=1.398-5.021 K_{t}+19.616 K_{t}^{3}+0.0135 S_{t}-0.0019 S_{t}^{2}$ \\
\hline & M29 & $K_{\mathrm{d}}=1.48-4.951 K_{t}+19.247 K_{t}^{3}-0.0101 S_{t}-5.276 e-05 S_{t}^{3}$ \\
\hline & M30 & $K_{\mathrm{d}}=1.041-5.116 K_{t}+20.136 K_{t}^{3}+0.11 S_{t}-0.0102 S_{t}^{2}+0.00022 S_{t}^{3}$ \\
\hline & M31 & $K_{\mathrm{d}}=1.437-4.912 K_{t+}+19.057 K_{t}^{3}-9.203 e-04 S_{t}^{2}-2.601 e-05 S_{t}^{3}$ \\
\hline & M32 & $K_{\mathrm{d}}=1.391-4.4841 K_{t}+16.87 K_{t}^{3}-0.0014 S_{t}^{2}$ \\
\hline & M33 & $K_{\mathrm{d}}=1.528-5.728 K_{t}+23.129 K_{t}^{3}-7.31 e-05 S_{t}^{3}$ \\
\hline & M34 & $K_{\mathrm{d}}=0.862-0.347-0.1 \log \left(S_{t}\right)$ \\
\hline & M35 & $K_{\mathrm{d}}=3.084-2.148 \exp \left(K_{t}\right)-1.87 e-08 \exp \left(S_{t}\right)$ \\
\hline & M37 & $K_{\mathrm{d}}=-0.2163+25.814\left(\frac{K_{t}}{S_{t}}\right)$ \\
\hline & M38 & $K_{\mathrm{d}}=-0.8485+86.043\left(\frac{K_{t}}{S_{t}}\right)-1409.06\left(\frac{K_{t}}{S_{t}}\right)^{2}$ \\
\hline & M39 & $K_{\mathrm{d}}=0.061+587.079\left(\frac{K_{t}}{S_{t}}\right)^{2}$ \\
\hline & M40 & $K_{\mathrm{d}}=0.155+1.714 e+04\left(\frac{K_{t}}{S_{t}}\right)^{3}$ \\
\hline
\end{tabular}


Table 2 (continued)
Models
Forms of the models

(b)

$K_{\mathrm{D}} \approx f\left(S_{t}\right)$

M41

M42

M43

M44

M45

$K_{\mathrm{D}} \approx f\left(K_{t}\right)$

M46

M47

M48

M49

M50

$K_{\mathrm{D}} \approx f\left(S_{t}, K_{t}\right)$
M51

M52

M53

M54

M55

M56

M57

M58

M59

M60

M61

M62

M63

M64

M65

M66

M67

M68

M69

M70

M71

M72

D73

M74

M75

M76

M77

M78

M79

M80
$K_{\mathrm{D}}=0.1869-0.0088 S_{t}$

$K_{\mathrm{D}}=0.0913+0.0069 S_{t}-0.0006 S_{t}^{2}$

$K_{\mathrm{D}}=0.0178-0.0261 S_{t}-2.27 e-03 S_{t}^{2}+4.522993 e-05 S_{t}^{3}$

$K_{\mathrm{D}}=0.337-0.1039 \log \left(S_{t}\right)$

$K_{\mathrm{D}}=0.082-6.736 e-09 \exp \left(S_{t}\right)$

$K_{\mathrm{D}}=0.2304-0.624 K_{t}$

$K_{\mathrm{D}}=0.3742-1.781 K_{t}+2.3171 K_{t}^{2}$

$K_{\mathrm{D}}=-1.4+19.719 K_{t}-84.085 K_{t}^{2}+115.184 K_{t}^{3}$

$K_{\mathrm{D}}=-0.141-0.155 \log \left(K_{t}\right)$

$K_{\mathrm{D}}=0.698-0.485 \exp \left(K_{t}\right)$

$K_{\mathrm{D}}=0.189-0.015 K_{t}-0.0087 S_{t}$

$K_{\mathrm{D}}=0.135-0.0064 K_{t}-0.00035 S_{t}^{2}$

$K_{\mathrm{D}}=0.119-0.0174 K_{t}-1.857 e-05 S_{t}^{3}$

$K_{\mathrm{D}}=0.089+0.7918 K_{t}-1.605 K_{t}^{2}-0.0088 S_{t}$

$K_{\mathrm{D}}=0.1160+0.1486 K_{t}-0.3093 K_{t}^{2}-0.00035 S_{t}^{2}$

$K_{\mathrm{D}}=0.1745-0.46 K_{t}+0.884 K_{t}^{2}-1.854 e-05 S_{t}^{3}$

$K_{\mathrm{D}}=0.1464-0.48 K_{t}+0.925 K_{t}^{2}+0.0079 S_{t}-0.0006 S_{t}^{2}$

$K_{\mathrm{D}}=0.074-0.526 K_{t}+1.019 K_{t}^{2}+0.028 S_{t}-2.39 e-03 S_{t}^{2} 4.77 e-05 S_{t}^{3}$

$K_{\mathrm{D}}=0.17-0.413 K_{t}+0.793 K_{t}^{2}-2.69 e-05 S_{t}^{2}-1.71 e-05 S_{t}^{3}$

$K_{\mathrm{D}}=0.828-8.168 K_{t}+34.445 K_{t}^{2}-48.093 K_{t}^{3}-0.0088 S_{t}$

$K_{\mathrm{D}}=2.121-24.497 K_{t}+97.204 K_{t}^{2}-127.98 K_{t}^{3}+1.005 e-02 S_{t}-7.63 e-04 S_{t}^{2}$

$K_{\mathrm{D}}=2.024-22.905 K_{t}+90.932 K_{t}^{2}-119.79 K_{t}^{3}+7.47 e-04 S_{t}-2.04 e-05 S_{t}^{3}$

$K_{\mathrm{D}}=2.404-29.725 K_{t}+117.98 K_{t}^{2}-155.36 K_{t}^{3}+4.96 e-02 S_{t}+0.0901 S_{t}^{2}-4.105 e-03 S_{t}^{3}$

$K_{\mathrm{D}}=1.989-22.456 K_{t}+89.222 K_{t}^{2}-117.636 K_{t}^{3}+3.77 e-05 S_{t}^{2}-2.086 e-05 S_{t}^{3}$

$K_{\mathrm{D}}=1.48-16.398 K_{t}+66.26 K_{t}^{2}-88.795 K_{t}^{3}-3.636 e-04 S_{t}^{2}$

$K_{\mathrm{D}}=1.945-21.937 K_{t} 87.272 K_{t}^{2}-115.205 K_{t}^{3}-1.89 e-05 S_{t}^{3}$

$K_{\mathrm{D}}=0.1208+0.4025 K_{t}-2.192 K_{t}^{3}-0.0088 S_{t}$

$K_{\mathrm{D}}=0.1233-0.2202 K_{t}+1.0782 K_{t}^{3}+0.0078 S_{t}-0.00066 S_{t}^{2}$

$K_{\mathrm{D}}=0.1516-0.2017 K_{t} 0.979 K_{t}^{3}-1.99 e-04 S_{t}-1.814 e-05 S_{t}^{3}$

$K_{\mathrm{D}}=0.049-0.2398 K+{ }_{t} 1.185 K_{t}^{3}+0.0278 S_{t}-2.36 e-03 S_{t}^{2}+4.7 e-05 S_{t}^{3}$

$K_{\mathrm{D}}=0.1494-0.188 K_{t}+0.9141 K_{t}^{3}-3.18 e-05 S_{t}^{2}-1.691711 e-05 S_{t}^{3}$

$K_{\mathrm{D}}=0.1193+0.0899 K_{t}-0.5084 K_{t}^{3}-0.00035 S_{t}^{2}$

$K_{\mathrm{D}}=0.1525-0.2168 K_{t}+1.0548 K_{t}^{3}-1.854 e-05 S_{t}^{3}$

$K_{\mathrm{D}}=0.3133-0.0108 \log \left(K_{t}\right)-0.1003 \log \left(S_{t}\right)$

$K_{\mathrm{D}}=0.4326-0.274 \exp \left(K_{t}\right)-5.091 e-09 \exp \left(S_{t}\right)$

$K_{\mathrm{D}}=-0.0382+5.6124\left(\frac{K_{t}}{S_{t}}\right)$

$K_{\mathrm{D}}=-0.2473+25.538\left(\frac{K_{t}}{S_{t}}\right)-466.17\left(\frac{K_{t}}{S_{t}}\right)^{2}$

$K_{\mathrm{D}}=6.058 e-02-17.445\left(\frac{K_{t}}{S_{t}}\right)+1501.46\left(\frac{K_{t}}{S_{t}}\right)^{2}-29502.33\left(\frac{K_{t}}{S_{t}}\right)^{3}$

$K_{\mathrm{D}}=0.0227+126.302\left(\frac{K_{t}}{S_{t}}\right)^{2}$

$K_{\mathrm{D}}=0.0433+3647.46\left(\frac{K_{t}}{S_{t}}\right)^{3}$ 

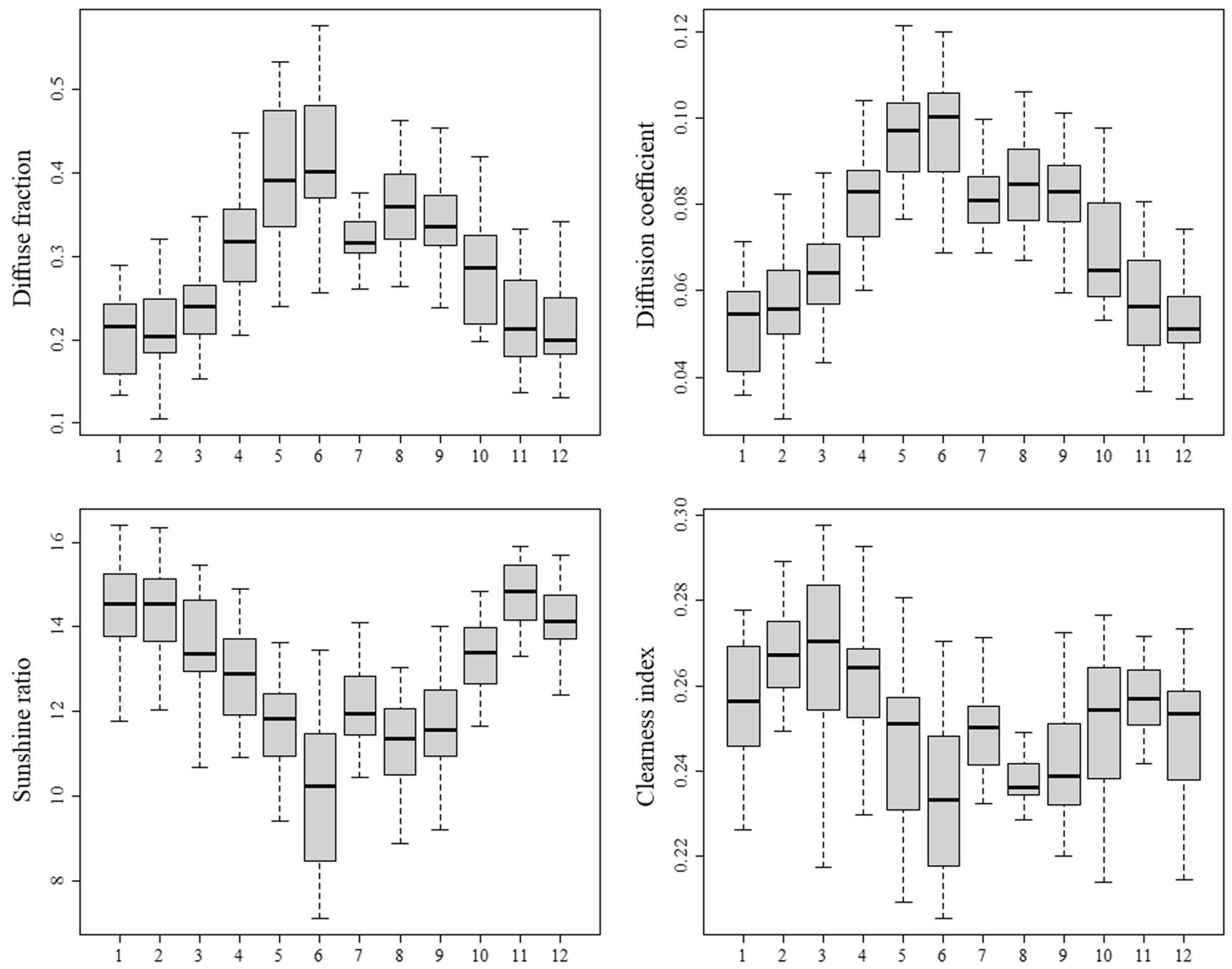

Fig. 2 Box plot of diffuse fraction (top left), diffusion coefficient (top right), sunshine ratio (bottom left) and clearness index (bottom right)

MAE, MARE, MAPE, RMSE, RMSRE, RRMSE and $t$-stat indicators should be closer to zero, but correlation coefficient $\left(R^{2}\right)$ should approach to 1 as closely as possible. From the statistical indicators, it can be seen that the estimated values of $k_{\mathrm{d}}$ and $K_{\mathrm{D}}$ are in good agreement with the measured values for the most models. The values of MBE are ranged from $-1.68 e^{-17}$ to $9.84 e^{-18}$ and $-1.81 e^{-18}$ to $1.06 e^{-18}$ for $k_{\mathrm{d}}$ and $K_{\mathrm{D}}$, respectively. We observed that the MBE values for the different models are very small which means that the proposed models slightly over predict the estimated values. The values of $t$-stats obtained for the all proposed models are significantly lower than the critical value. In terms of MBE and $t$-stats, the models M40 for $k_{\mathrm{d}}$ and $\mathrm{M} 65$ for $K_{\mathrm{D}}$ show the lowest errors. However, in terms of MAE, MARE, MAPE, RMSE, RMSRE, and RRMSE, the cubic model for both $k_{\mathrm{d}}$ and $K_{\mathrm{D}}$ (M23 for $k_{\mathrm{d}}$ and M63 for $K_{\mathrm{D}}$ ) shows excellent accuracy since the MAE,
MARE, MAPE, RMSE, RMSRE, and RRMSE values are the lowest. The correlation coefficient $\left(R^{2}\right)$ is observed to be the highest for M23 $\left(k_{\mathrm{d}}\right)$ and M63 $\left(K_{\mathrm{D}}\right)$ among all the models proposed, which means that the estimated and observed data from the cubic equations shows a maximum closeness.

The results of the statistical indicators show that the estimated diffuse fraction and diffuse coefficient values from the different proposed models are close to each other. Since not all the statistical indicators are in favor of a model, more appropriate combined statistical indicators which can yield a comparative performance of the proposed models need to be established. In this way, we used Global Performance Indicator (GPI) that represents multiplication of all used statistical indicators (Said and Dickey 1983; Despotovic et al. 2015; Jamil and Abid 2018). 

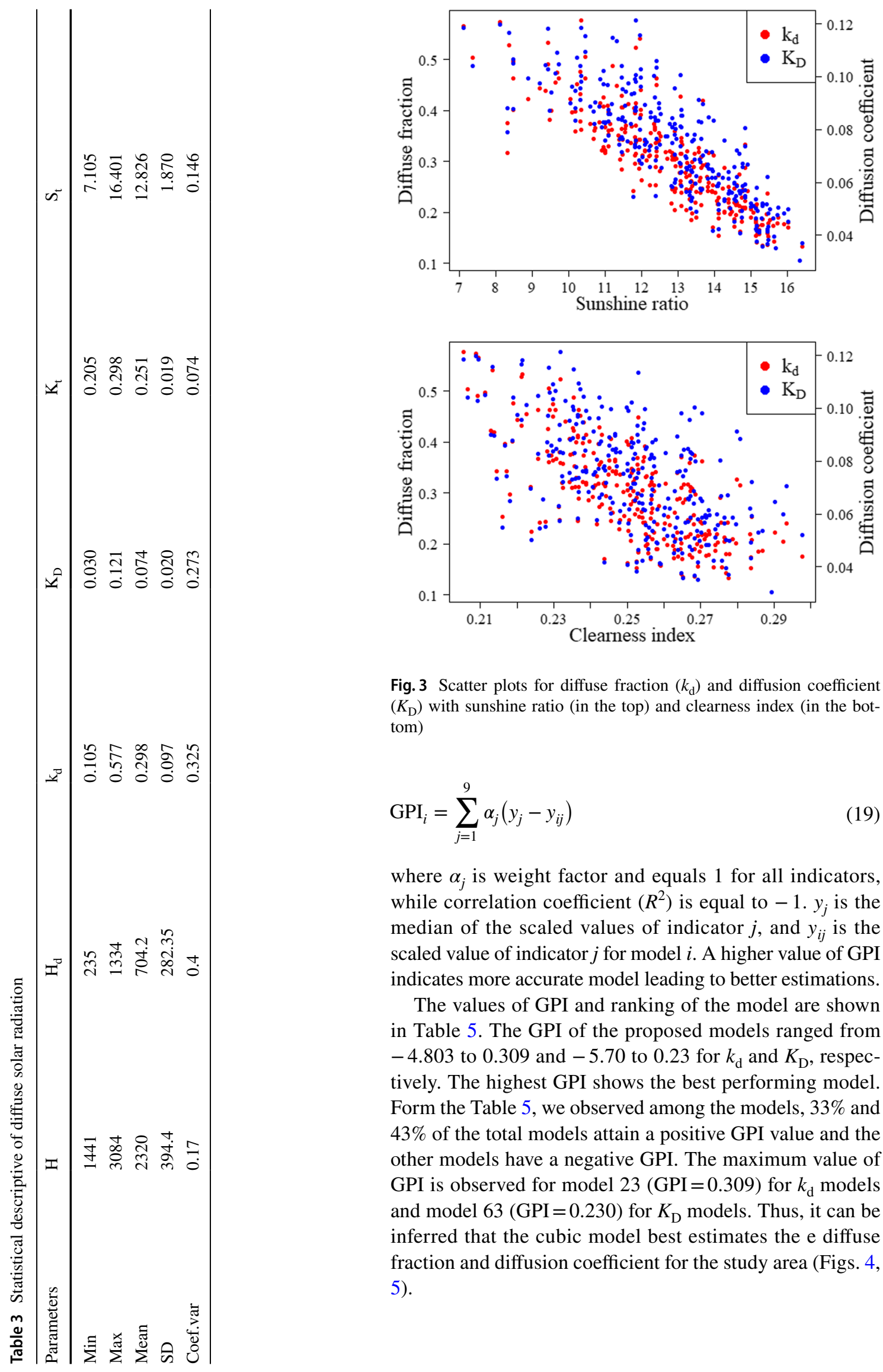

Fig. 3 Scatter plots for diffuse fraction $\left(k_{\mathrm{d}}\right)$ and diffusion coefficient $\left(K_{\mathrm{D}}\right)$ with sunshine ratio (in the top) and clearness index (in the bottom)

$\mathrm{GPI}_{i}=\sum_{j=1}^{9} \alpha_{j}\left(y_{j}-y_{i j}\right)$

where $\alpha_{j}$ is weight factor and equals 1 for all indicators, while correlation coefficient $\left(R^{2}\right)$ is equal to $-1 . y_{j}$ is the median of the scaled values of indicator $j$, and $y_{i j}$ is the scaled value of indicator $j$ for model $i$. A higher value of GPI indicates more accurate model leading to better estimations.

The values of GPI and ranking of the model are shown in Table 5. The GPI of the proposed models ranged from -4.803 to 0.309 and -5.70 to 0.23 for $k_{\mathrm{d}}$ and $K_{\mathrm{D}}$, respectively. The highest GPI shows the best performing model. Form the Table 5, we observed among the models, 33\% and $43 \%$ of the total models attain a positive GPI value and the other models have a negative GPI. The maximum value of GPI is observed for model $23(\mathrm{GPI}=0.309)$ for $k_{\mathrm{d}}$ models and model $63(\mathrm{GPI}=0.230)$ for $K_{\mathrm{D}}$ models. Thus, it can be inferred that the cubic model best estimates the e diffuse fraction and diffusion coefficient for the study area (Figs. 4, $5)$. 
Table 4 Results of statistical indicators for all proposed models

\begin{tabular}{|c|c|c|c|c|c|c|c|c|c|}
\hline Models & MBE & MAE & MARE & MAPE & RMSE & RMSRE & RRMSE & $\mathrm{R}^{2}$ & t-stat \\
\hline & \multicolumn{9}{|l|}{$k_{\mathrm{d}}$} \\
\hline M1 & $5.62 \mathrm{E}-19$ & 0.0362 & 0.1233 & 12.6151 & 0.0493 & 0.1638 & 9.0407 & 0.7385 & $1.89 \mathrm{E}-16$ \\
\hline M2 & $2.75 \mathrm{E}-18$ & 0.0362 & 0.1236 & 12.5850 & 0.0489 & 0.1632 & 8.9641 & 0.7429 & $9.32 \mathrm{E}-16$ \\
\hline M3 & $2.06 \mathrm{E}-18$ & 0.0362 & 0.1232 & 12.5533 & 0.0488 & 0.1627 & 8.9533 & 0.7435 & $7.00 \mathrm{E}-16$ \\
\hline M4 & $-7.50 \mathrm{E}-19$ & 0.0376 & 0.1284 & 13.2475 & 0.0512 & 0.1701 & 9.3866 & 0.7181 & $2.43 E-16$ \\
\hline M5 & $-1.70 \mathrm{E}-18$ & 0.0567 & 0.2223 & 20.9577 & 0.0734 & 0.4064 & 13.4539 & 0.4209 & $3.84 \mathrm{E}-16$ \\
\hline M6 & $1.20 \mathrm{E}-18$ & 0.0541 & 0.1879 & 20.4662 & 0.0667 & 0.2347 & 12.2255 & 0.5218 & $2.98 \mathrm{E}-16$ \\
\hline M7 & $2.32 \mathrm{E}-18$ & 0.0530 & 0.1819 & 20.0034 & 0.0659 & 0.2260 & 12.0829 & 0.5329 & $5.83 \mathrm{E}-16$ \\
\hline M8 & $-5.51 \mathrm{E}-18$ & 0.0530 & 0.1815 & 19.9259 & 0.0658 & 0.2255 & 12.0668 & 0.5342 & $1.39 \mathrm{E}-15$ \\
\hline M9 & $-1.68 \mathrm{E}-17$ & 0.0537 & 0.1856 & 20.2942 & 0.0663 & 0.2306 & 12.1502 & 0.5277 & $4.20 \mathrm{E}-15$ \\
\hline M10 & $-1.20 \mathrm{E}-18$ & 0.0542 & 0.1886 & 20.5140 & 0.0668 & 0.2360 & 12.2491 & 0.5200 & $2.97 \mathrm{E}-16$ \\
\hline M11 & $9.84 \mathrm{E}-18$ & 0.0354 & 0.1206 & 12.3883 & 0.0462 & 0.1538 & 8.4745 & 0.7702 & $3.53 E-15$ \\
\hline M12 & $-1.23 \mathrm{E}-18$ & 0.0355 & 0.1216 & 12.4322 & 0.0458 & 0.1534 & 8.3934 & 0.7746 & $4.46 \mathrm{E}-16$ \\
\hline M13 & $1.78 \mathrm{E}-18$ & 0.0362 & 0.1257 & 12.7949 & 0.0462 & 0.1575 & 8.4617 & 0.7709 & $6.39 \mathrm{E}-16$ \\
\hline M14 & $-1.59 \mathrm{E}-19$ & 0.0354 & 0.1207 & 12.4246 & 0.0461 & 0.1532 & 8.4524 & 0.7714 & $5.71 \mathrm{E}-17$ \\
\hline M15 & $3.08 \mathrm{E}-18$ & 0.0353 & 0.1204 & 12.3687 & 0.0454 & 0.1514 & 8.3298 & 0.7780 & $1.13 \mathrm{E}-15$ \\
\hline M16 & $5.05 \mathrm{E}-18$ & 0.0355 & 0.1218 & 12.4955 & 0.0455 & 0.1529 & 8.3430 & 0.7773 & $1.84 \mathrm{E}-15$ \\
\hline M17 & $-1.12 \mathrm{E}-18$ & 0.0353 & 0.1209 & 12.4055 & 0.0454 & 0.1516 & 8.3187 & 0.7786 & $4.11 \mathrm{E}-16$ \\
\hline M18 & $-4.40 \mathrm{E}-18$ & 0.0353 & 0.1205 & 12.3795 & 0.0453 & 0.1511 & 8.3063 & 0.7793 & $1.61 \mathrm{E}-15$ \\
\hline M19 & $3.00 \mathrm{E}-18$ & 0.0353 & 0.1208 & 12.4036 & 0.0454 & 0.1516 & 8.3221 & 0.7784 & $1.10 \mathrm{E}-15$ \\
\hline M20 & $1.42 \mathrm{E}-18$ & 0.0351 & 0.1198 & 12.3273 & 0.0460 & 0.1531 & 8.4259 & 0.7729 & $5.13 E-16$ \\
\hline M21 & $6.45 \mathrm{E}-18$ & 0.0348 & 0.1195 & 12.2581 & 0.0450 & 0.1519 & 8.2431 & 0.7826 & $2.38 \mathrm{E}-15$ \\
\hline M22 & $7.37 \mathrm{E}-19$ & 0.0348 & 0.1198 & 12.2837 & 0.0450 & 0.1521 & 8.2558 & 0.7819 & $2.71 \mathrm{E}-16$ \\
\hline M23 & $2.96 \mathrm{E}-19$ & 0.0347 & 0.1184 & 12.1390 & 0.0447 & 0.1510 & 8.2026 & 0.7847 & $1.10 \mathrm{E}-16$ \\
\hline M24 & $3.11 \mathrm{E}-18$ & 0.0348 & 0.1195 & 12.2610 & 0.0450 & 0.1519 & 8.2528 & 0.7821 & $1.14 \mathrm{E}-15$ \\
\hline M25 & $2.95 \mathrm{E}-19$ & 0.0347 & 0.1187 & 12.1870 & 0.0451 & 0.1512 & 8.2727 & 0.7810 & $1.08 \mathrm{E}-16$ \\
\hline M26 & $-1.34 \mathrm{E}-18$ & 0.0349 & 0.1205 & 12.3476 & 0.0451 & 0.1530 & 8.2615 & 0.7816 & $4.94 \mathrm{E}-16$ \\
\hline M27 & $-1.97 \mathrm{E}-18$ & 0.0354 & 0.1208 & 12.4266 & 0.0461 & 0.1532 & 8.4540 & 0.7713 & $7.08 \mathrm{E}-16$ \\
\hline M28 & $2.46 \mathrm{E}-18$ & 0.0353 & 0.1209 & 12.4123 & 0.0454 & 0.1517 & 8.3238 & 0.7783 & $8.99 \mathrm{E}-16$ \\
\hline M29 & $-7.86 \mathrm{E}-18$ & 0.0354 & 0.1210 & 12.4188 & 0.0454 & 0.1518 & 8.3299 & 0.7780 & $2.87 \mathrm{E}-15$ \\
\hline M30 & $-1.78 \mathrm{E}-18$ & 0.0354 & 0.1206 & 12.3866 & 0.0453 & 0.1512 & 8.3122 & 0.7790 & $6.51 \mathrm{E}-16$ \\
\hline M31 & $4.89 \mathrm{E}-18$ & 0.0353 & 0.1209 & 12.4092 & 0.0454 & 0.1517 & 8.3270 & 0.7782 & $1.78 \mathrm{E}-15$ \\
\hline M32 & $-1.62 \mathrm{E}-19$ & 0.0353 & 0.1205 & 12.3750 & 0.0455 & 0.1515 & 8.3338 & 0.7778 & $5.91 \mathrm{E}-17$ \\
\hline M33 & $1.15 \mathrm{E}-18$ & 0.0356 & 0.1220 & 12.5096 & 0.0456 & 0.1530 & 8.3495 & 0.7770 & $4.20 \mathrm{E}-16$ \\
\hline M34 & $5.97 \mathrm{E}-18$ & 0.0365 & 0.1248 & 12.9129 & 0.0477 & 0.1587 & 8.7395 & 0.7556 & $2.08 \mathrm{E}-15$ \\
\hline M35 & $7.60 \mathrm{E}-18$ & 0.0472 & 1.7216 & 17.5514 & 0.0586 & 25.4900 & 10.7396 & 0.6310 & $2.15 \mathrm{E}-15$ \\
\hline M36 & $-1.01 \mathrm{E}-18$ & 0.0550 & 0.1912 & 20.5253 & 0.0730 & 0.2586 & 13.3757 & 0.4276 & $2.28 \mathrm{E}-16$ \\
\hline M37 & $2.52 \mathrm{E}-18$ & 0.0532 & 0.1872 & 19.7012 & 0.0717 & 0.2677 & 13.1441 & 0.4473 & $5.82 \mathrm{E}-16$ \\
\hline M38 & $-2.01 \mathrm{E}-18$ & 0.0536 & 0.1873 & 19.8362 & 0.0713 & 0.2579 & 13.0705 & 0.4534 & $4.68 \mathrm{E}-16$ \\
\hline M39 & $1.98 \mathrm{E}-19$ & 0.0563 & 0.1948 & 21.1221 & 0.0742 & 0.2586 & 13.6087 & 0.4075 & $4.42 \mathrm{E}-17$ \\
\hline \multirow[t]{2}{*}{ M40 } & $1.96 \mathrm{E}-19$ & 0.0583 & 0.2002 & 21.9386 & 0.0760 & 0.2609 & 13.9277 & 0.3794 & $4.28 \mathrm{E}-17$ \\
\hline & \multicolumn{9}{|l|}{$K_{\mathrm{D}}$} \\
\hline M41 & $1.06 \mathrm{E}-18$ & 0.0088 & 0.1214 & 12.4840 & 0.0113 & 0.1552 & 4.1724 & 0.6805 & $1.55 \mathrm{E}-15$ \\
\hline M42 & $1.22 \mathrm{E}-19$ & 0.0086 & 0.1198 & 12.2569 & 0.0110 & 0.1510 & 4.0407 & 0.7003 & $1.85 \mathrm{E}-16$ \\
\hline M43 & $-4.73 E-19$ & 0.0086 & 0.1196 & 12.2411 & 0.0109 & 0.1507 & 4.0369 & 0.7009 & $7.16 \mathrm{E}-16$ \\
\hline M44 & $-5.60 \mathrm{E}-19$ & 0.0092 & 0.1268 & 13.1286 & 0.0119 & 0.1624 & 4.3750 & 0.6487 & $7.83 \mathrm{E}-16$ \\
\hline M45 & $4.23 \mathrm{E}-19$ & 0.0121 & 0.3212 & 17.9279 & 0.0149 & 2.2351 & 5.5106 & 0.4426 & $4.70 \mathrm{E}-16$ \\
\hline M46 & $2.88 \mathrm{E}-19$ & 0.0133 & 0.1836 & 20.1637 & 0.0163 & 0.2277 & 6.0281 & 0.3330 & $2.92 \mathrm{E}-16$ \\
\hline M47 & $-4.01 \mathrm{E}-20$ & 0.0132 & 0.1822 & 20.0393 & 0.0163 & 0.2262 & 6.0168 & 0.3355 & $4.08 \mathrm{E}-17$ \\
\hline M48 & $1.42 \mathrm{E}-19$ & 0.0132 & 0.1816 & 19.9307 & 0.0163 & 0.2255 & 5.9992 & 0.3394 & $1.45 \mathrm{E}-16$ \\
\hline M49 & $-8.45 \mathrm{E}-19$ & 0.0132 & 0.1827 & 20.0859 & 0.0163 & 0.2268 & 6.0201 & 0.3348 & $8.59 \mathrm{E}-16$ \\
\hline M50 & $8.89 \mathrm{E}-19$ & 0.0133 & 0.1839 & 20.1882 & 0.0164 & 0.2279 & 6.0314 & 0.3323 & $9.02 \mathrm{E}-16$ \\
\hline M51 & $7.51 \mathrm{E}-19$ & 0.0088 & 0.1216 & 12.5056 & 0.0113 & 0.1551 & 4.1717 & 0.6806 & $1.10 \mathrm{E}-15$ \\
\hline M52 & $-1.84 \mathrm{E}-19$ & 0.0086 & 0.1194 & 12.2431 & 0.0110 & 0.1514 & 4.0664 & 0.6965 & $2.77 \mathrm{E}-16$ \\
\hline M53 & $-2.31 \mathrm{E}-19$ & 0.0087 & 0.1202 & 12.3079 & 0.0110 & 0.1513 & 4.0442 & 0.6998 & $3.49 \mathrm{E}-16$ \\
\hline M54 & $4.98 \mathrm{E}-19$ & 0.0088 & 0.1211 & 12.4348 & 0.0113 & 0.1550 & 4.1640 & 0.6817 & $7.32 \mathrm{E}-16$ \\
\hline
\end{tabular}


Table 4 (continued)

\begin{tabular}{|c|c|c|c|c|c|c|c|c|c|}
\hline Models & MBE & MAE & MARE & MAPE & RMSE & RMSRE & RRMSE & $\mathrm{R}^{2}$ & t-stat \\
\hline M55 & $9.65 \mathrm{E}-20$ & 0.0086 & 0.1194 & 12.2337 & 0.0110 & 0.1515 & 4.0661 & 0.6965 & $1.45 \mathrm{E}-16$ \\
\hline M56 & $-2.52 \mathrm{E}-20$ & 0.0087 & 0.1201 & 12.3066 & 0.0110 & 0.1510 & 4.0417 & 0.7002 & $3.81 \mathrm{E}-17$ \\
\hline M57 & $-3.03 E-19$ & 0.0087 & 0.1199 & 12.2913 & 0.0109 & 0.1508 & 4.0378 & 0.7007 & $4.59 \mathrm{E}-16$ \\
\hline M58 & $-7.03 \mathrm{E}-19$ & 0.0087 & 0.1197 & 12.2747 & 0.0109 & 0.1505 & 4.0335 & 0.7014 & $1.07 \mathrm{E}-15$ \\
\hline M59 & $-1.81 \mathrm{E}-18$ & 0.0087 & 0.1200 & 12.2963 & 0.0110 & 0.1510 & 4.0416 & 0.7002 & $2.73 \mathrm{E}-15$ \\
\hline M60 & $1.23 \mathrm{E}-19$ & 0.0087 & 0.1209 & 12.4151 & 0.0113 & 0.1550 & 4.1597 & 0.6824 & $1.80 \mathrm{E}-16$ \\
\hline M61 & $-7.23 E-19$ & 0.0086 & 0.1187 & 12.1584 & 0.0109 & 0.1506 & 4.0085 & 0.7051 & $1.10 \mathrm{E}-15$ \\
\hline M62 & $-9.15 \mathrm{E}-20$ & 0.0086 & 0.1189 & 12.1826 & 0.0109 & 0.1509 & 4.0158 & 0.7040 & $1.39 \mathrm{E}-16$ \\
\hline M63 & $-3.28 \mathrm{E}-19$ & 0.0085 & 0.1179 & 12.0803 & 0.0108 & 0.1500 & 3.9940 & 0.7072 & $5.02 \mathrm{E}-16$ \\
\hline M64 & $-7.76 \mathrm{E}-19$ & 0.0086 & 0.1188 & 12.1755 & 0.0109 & 0.1509 & 4.0163 & 0.7039 & $1.18 \mathrm{E}-15$ \\
\hline M65 & 0.00 & 0.0086 & 0.1184 & 12.1320 & 0.0110 & 0.1512 & 4.0512 & 0.6988 & 0.000 \\
\hline M66 & $-7.76 \mathrm{E}-19$ & 0.0086 & 0.1187 & 12.1618 & 0.0109 & 0.1507 & 4.0166 & 0.7039 & $1.18 \mathrm{E}-15$ \\
\hline M67 & $-3.75 E-19$ & 0.0088 & 0.1211 & 12.4329 & 0.0113 & 0.1550 & 4.1636 & 0.6818 & $5.51 \mathrm{E}-16$ \\
\hline M68 & $-3.53 \mathrm{E}-19$ & 0.0087 & 0.1199 & 12.2903 & 0.0110 & 0.1508 & 4.0383 & 0.7007 & $5.35 \mathrm{E}-16$ \\
\hline M69 & $6.78 \mathrm{E}-19$ & 0.0087 & 0.1200 & 12.3011 & 0.0110 & 0.1510 & 4.0421 & 0.7001 & $1.03 \mathrm{E}-15$ \\
\hline M70 & $6.31 \mathrm{E}-19$ & 0.0087 & 0.1197 & 12.2746 & 0.0109 & 0.1505 & 4.0342 & 0.7013 & $9.56 \mathrm{E}-16$ \\
\hline M71 & $6.07 \mathrm{E}-19$ & 0.0087 & 0.1200 & 12.2949 & 0.0110 & 0.1510 & 4.0420 & 0.7001 & $9.18 \mathrm{E}-16$ \\
\hline M72 & $-2.06 \mathrm{E}-19$ & 0.0086 & 0.1194 & 12.2309 & 0.0110 & 0.1515 & 4.0659 & 0.6966 & $3.09 \mathrm{E}-16$ \\
\hline M73 & $-6.30 \mathrm{E}-19$ & 0.0087 & 0.1201 & 12.3073 & 0.0110 & 0.1511 & 4.0422 & 0.7001 & $9.54 \mathrm{E}-16$ \\
\hline M74 & $-2.82 \mathrm{E}-20$ & 0.0092 & 0.1274 & 13.2006 & 0.0118 & 0.1622 & 4.3697 & 0.6495 & $3.95 \mathrm{E}-17$ \\
\hline M75 & $-5.05 \mathrm{E}-19$ & 0.0113 & 0.2173 & 16.8329 & 0.0138 & 0.7882 & 5.1027 & 0.5221 & $6.05 \mathrm{E}-16$ \\
\hline M76 & $2.03 \mathrm{E}-19$ & 0.0113 & 0.1575 & 16.6378 & 0.0146 & 0.2070 & 5.3764 & 0.4694 & $2.31 \mathrm{E}-16$ \\
\hline M77 & $-8.06 \mathrm{E}-19$ & 0.0104 & 0.1480 & 15.2410 & 0.0139 & 0.2115 & 5.1171 & 0.5194 & $9.63 \mathrm{E}-16$ \\
\hline M78 & $-7.76 \mathrm{E}-19$ & 0.0104 & 0.1483 & 15.3289 & 0.0138 & 0.2062 & 5.1023 & 0.5221 & $9.30 \mathrm{E}-16$ \\
\hline M79 & $3.28 \mathrm{E}-19$ & 0.0117 & 0.1625 & 17.3442 & 0.0150 & 0.2098 & 5.5332 & 0.4380 & $3.63 \mathrm{E}-16$ \\
\hline M80 & $3.28 \mathrm{E}-19$ & 0.0122 & 0.1685 & 18.1671 & 0.0155 & 0.2144 & 5.7230 & 0.3988 & $3.51 \mathrm{E}-16$ \\
\hline
\end{tabular}

Table 5 The global

performance indicator (GPI) and ranking for all proposed models

\begin{tabular}{llllrrlrrrrr}
\hline Models & GPI & Rank & Models & GPI & Rank & Models & GPI & Rank & Models & GPI & Rank \\
\hline & \multicolumn{1}{l}{$k_{\mathrm{d}}$} & & & & & & & & & & \\
M1 & -0.245 & 20 & M11 & -1.058 & 28 & M21 & -0.504 & 25 & M31 & -0.382 & 22 \\
M2 & -0.462 & 24 & M12 & 0.128 & 9 & M22 & 0.206 & 5 & M32 & 0.225 & 3 \\
M3 & -0.373 & 21 & M13 & -0.136 & 16 & M23 & 0.309 & 1 & M33 & 0.054 & 11 \\
M4 & -0.507 & 26 & M14 & 0.157 & 7 & M24 & -0.088 & 15 & M34 & -0.796 & 27 \\
M5 & -4.321 & 37 & M15 & -0.150 & 18 & M25 & 0.269 & 2 & M35 & -4.803 & 40 \\
M6 & -3.543 & 32 & M16 & -0.427 & 23 & M26 & 0.217 & 4 & M36 & -4.125 & 36 \\
M7 & -3.480 & 31 & M17 & 0.178 & 6 & M27 & 0.067 & 10 & M37 & -4.052 & 35 \\
M8 & -3.361 & 29 & M18 & 0.022 & 13 & M28 & -0.078 & 14 & M38 & -3.843 & 34 \\
M9 & -3.729 & 33 & M19 & -0.143 & 17 & M29 & -0.168 & 19 & M39 & -4.378 & 38 \\
M10 & -3.476 & 30 & M20 & 0.024 & 12 & M30 & 0.149 & 8 & M40 & -4.727 & 39 \\
& $K_{\mathrm{D}}$ & & & & & & & & & & \\
M41 & -1.009 & 28 & M51 & -0.744 & 27 & M61 & 0.109 & 9 & M71 & -0.380 & 21 \\
M42 & 0.069 & 14 & M52 & 0.110 & 7 & M62 & 0.224 & 2 & M72 & 0.109 & 8 \\
M43 & 0.090 & 11 & M53 & 0.113 & 5 & M63 & 0.230 & 1 & M73 & 0.036 & 17 \\
M44 & -0.634 & 26 & M54 & -0.489 & 24 & M64 & 0.083 & 13 & M74 & -0.561 & 25 \\
M45 & -5.679 & 39 & M55 & 0.063 & 15 & M65 & 0.204 & 3 & M75 & -3.290 & 32 \\
M46 & -5.257 & 38 & M56 & 0.160 & 4 & M66 & 0.086 & 12 & M76 & -3.203 & 31 \\
M47 & -4.994 & 35 & M57 & 0.113 & 6 & M67 & -0.117 & 18 & M77 & -2.334 & 30 \\
M48 & -5.046 & 37 & M58 & 0.039 & 16 & M68 & 0.102 & 10 & M78 & -2.331 & 29 \\
M49 & -5.031 & 36 & M59 & -0.201 & 20 & M69 & -0.446 & 23 & M79 & -3.507 & 33 \\
M50 & -5.703 & 40 & M60 & -0.142 & 19 & M70 & -0.387 & 22 & M80 & -4.029 & 34 \\
\hline
\end{tabular}


Model 23: $\mathrm{k}_{\mathrm{d}}=\mathrm{f}\left(\mathrm{S}_{\mathrm{t}}, \mathrm{K}_{\mathrm{t}}\right)$
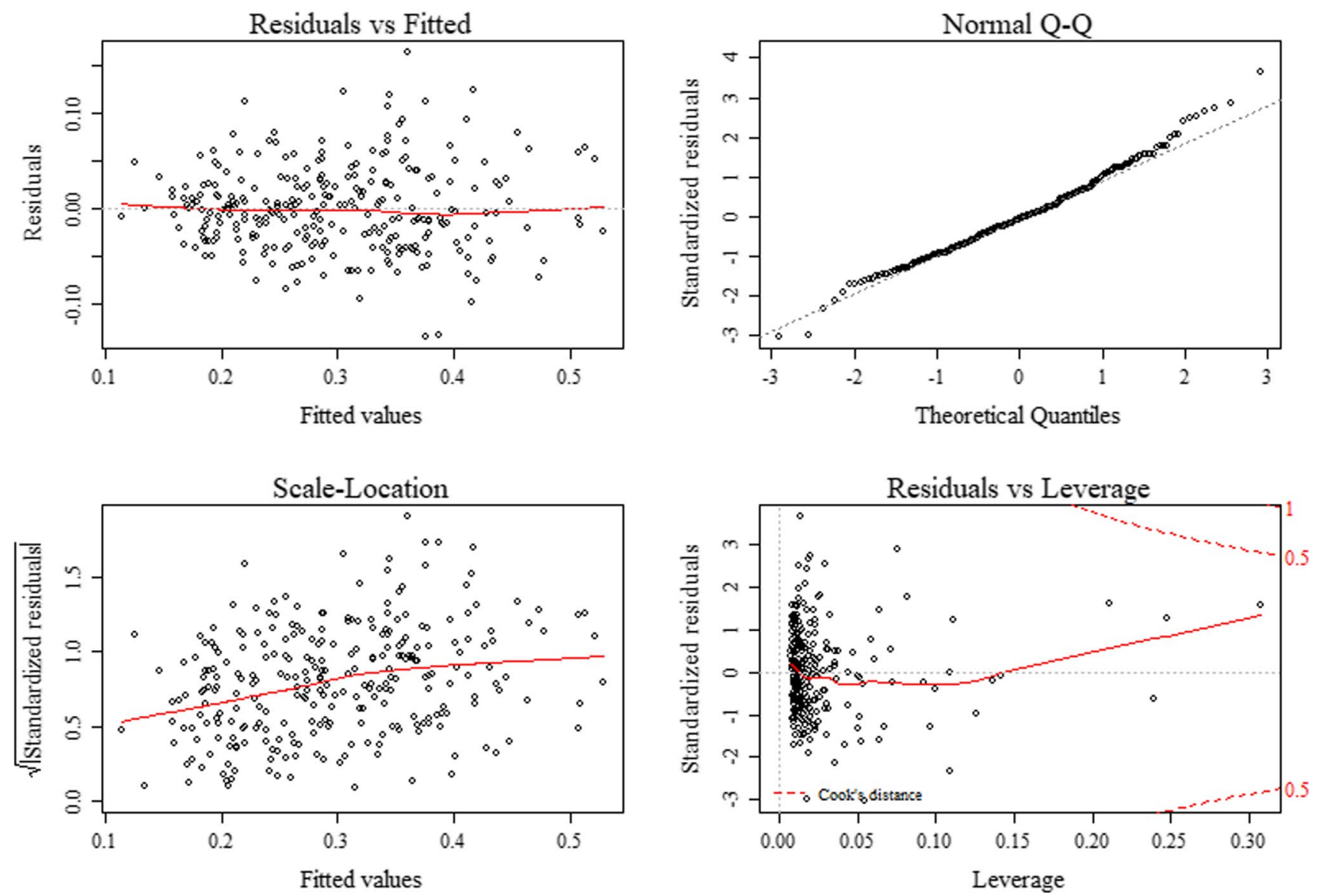

Fig. 4 Diagnostic plots of the best model of $k_{\mathrm{d}}$ (M23)

\section{Conclusion}

In this study, solar radiation data was used to evaluation the diffuse fraction and diffusion coefficient using sunshine ratio and clearness index as predictors in Tamanrasset station, Algeria. The results show that the high values of $k_{\mathrm{d}}$ and $K_{\mathrm{D}}$ are observed between the months of April to September; however, those of $S_{t}$ and $K_{t}$ are observed between

the months of January to December. Significant negative correlation between $k_{\mathrm{d}}-S_{t}, k_{\mathrm{d}}-K_{t}, K_{\mathrm{D}}-S_{t}$, and $K_{\mathrm{D}} K_{t}$. Forty models are proposed in order to estimate the diffuse fraction and diffusion coefficient using sunshine ratio and clearness index as predictors. Based on the values of different statistical indicators and GPI, the best models for diffuse fraction and diffusion coefficient are models 23 and 63, respectively. 

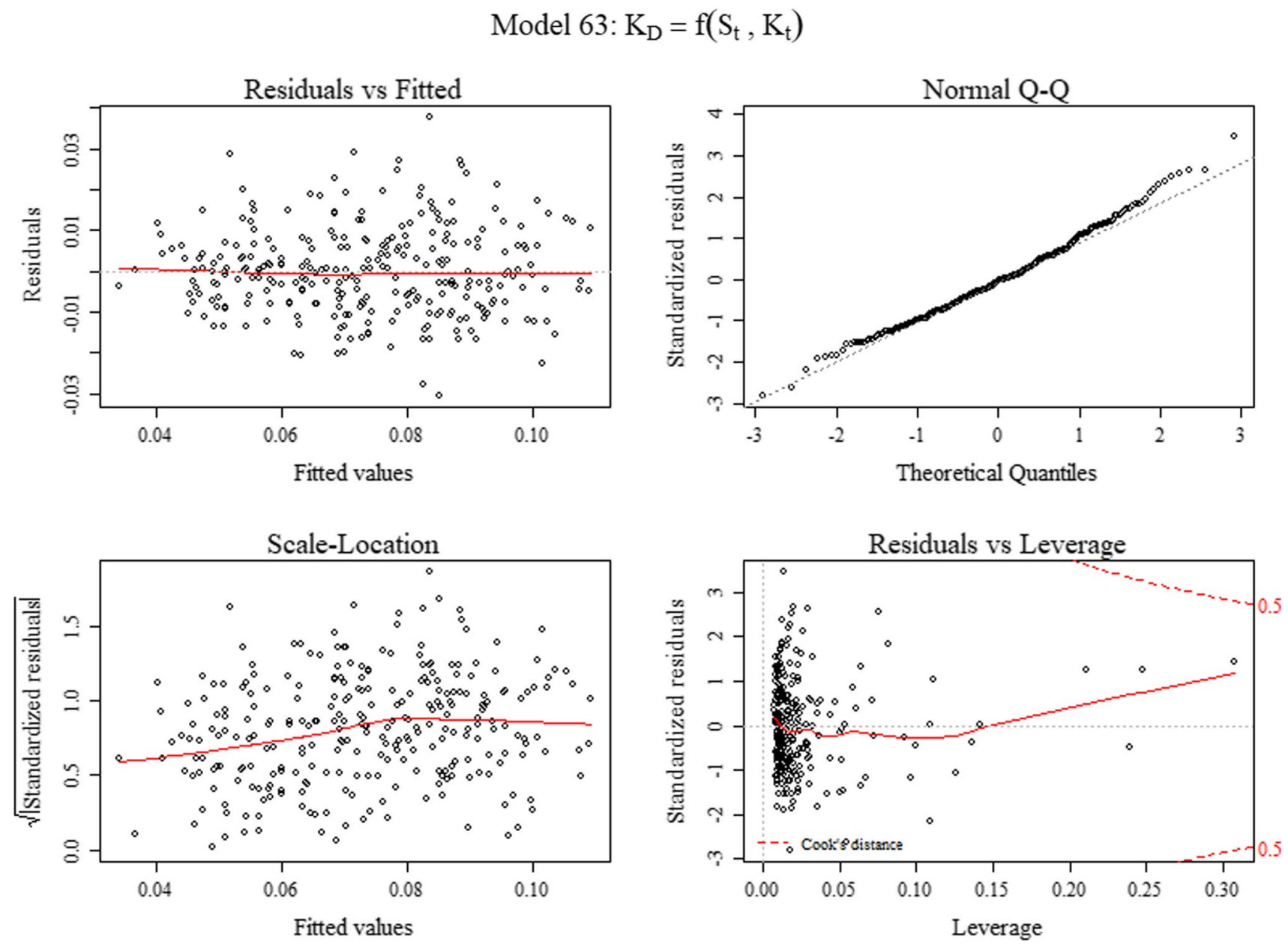

Fig. 5 Diagnostic plots of the best model of $K_{\mathrm{D}}$ (M63)

\section{Compliance with ethical standards}

Conflict of interest The authors declare that there is no conflict of interest in the publication of this article.

Open Access This article is licensed under a Creative Commons Attribution 4.0 International License, which permits use, sharing, adaptation, distribution and reproduction in any medium or format, as long as you give appropriate credit to the original author(s) and the source, provide a link to the Creative Commons licence, and indicate if changes were made. The images or other third party material in this article are included in the article's Creative Commons licence, unless indicated otherwise in a credit line to the material. If material is not included in the article's Creative Commons licence and your intended use is not permitted by statutory regulation or exceeds the permitted use, you will need to obtain permission directly from the copyright holder. To view a copy of this licence, visit http://creativecommons.org/licenses/by/4.0/.

\section{References}

Bailek N, Bouchouicha K, Al-Mostafa Z, El-Shimy M, Aoun N, Slimani A, Al- Shehri S (2017) A new empirical model for forecasting

the diffuse solar radiation over Sahara in the Algerian Big South. Renew Energy. https://doi.org/10.1016/j.renene.2017.10.081

Bakirci K (2009) Correlations for estimation of daily global solar radiation with hours of bright sunshine in Turkey. Energy 34(4):485-501

Bakirci K (2015) Models for the estimation of diffuse solar radiation for typical cities in Turkey. Energy 82:827-838

Bouchouicha K, Razagui A, Bachari NEI, Aoun N (2015) Mapping and geospatial analysis of solar resource in Algeria. Int J Energy Environ Econ 23:735

BoudgheneStambouli A (2011) Algerian renewable energy assessment: the challenge of sustainability. Energy Policy 39:4507-4519

De Miguel A, Bilbao J, Aguiar R, Kambezidis H, Negro E (2001) Diffuse solar irradiation model evaluation in the north Mediterranean belt area. Sol Energy 70(2001):143-153

Despotovic M, Nedic V, Despotovic D, Cvetanovic S (2015) Review and statistical analysis of different global solar radiation sunshine models. Renew Sustain Energy Rev 56:1869-1880

Erbs D, Klein S, Duffie J (1982) Estimation of the diffuse radiation fraction for hourly, daily and monthly-average global radiation. Sol Energy 28:293-302

Hua S, Shengyuan T, Fenxian S (2002) Models to separate daily diffuse radiation from daily total radiation for energy consumption analysis of air conditioning system. J Chongqing Univ (Nat Sci Ed) 25:73-76 
Hussain M, Rahman L, Mohibur Rahman M (1999) Techniques to obtain improved predictions of global radiation from sunshine duration. Renew Energy. 18:263-275

Iqbal M (1979) A study of Canadian diffuse and total solar radiation data-I monthly average daily horizontal radiation. Sol Energy 22:81-86

Jamil B, Abid T (2018) Siddiqui. Estimation of monthly mean diffuse solar radiation over India: performance of two variable models under different climatic zones. Sustain Energy Technol Assess 25:161-180

Klein SA (1977) Calculation of monthly average insolation on tilted surfaces. Sol Energy 19:325-330

Kuo CW, Chang WC, Chang KC (2014) Modeling the hourly solar diffuse fraction in Taiwan. Renew Energy 66:56-61

Lam JC, Li DHW (1996) Correlation between global solar radiation and its direct and diffuse components. Build Environ 31:527-535

Li H, Bu X, Lian Y, Zhao L, Ma W (2012) Further investigation of empirically derived models with multiple predictors in estimating monthly average daily diffuse solar radiation over China. Renew Energy 44:469-473

Liu Y, Zhou Y, Wang D, Wang Y, Li Y, Zhu Y (2017) Classification of solar radiation zones and general models for estimating the daily global solar radiation on horizontal surfaces in China. Energy Convers Manag 154:168-179

Mecibah MS, Boukelia TE, Tahtah R, Gairaa K (2014) Introducing the best model for estimation the monthly mean daily global solar radiation on a horizontal surface (case study: Algeria). Renew Sustain Energy Rev 36:194-202

Orgill JF, Hollands KGT (1977) Correlation equation for hourly diffuse radiation on a horizontal surface. Sol Energy 19:357-359

Paliatsos A, Kambezidis H, Antoniou A (2003) Diffuse solar irradiation at a location in the Balkan Peninsula. Renew Energy 28:2147-2156

Reindl DT, Beckman WA, Duffie JA (1990) Diffuse fraction correlations. Sol Energy 45:1-7

Sabbagh J, Sayigh A, El-Salam E (1977) Estimation of the total solar radiation from meteorological data. Sol Energy 251(19):307-311

Said SE, Dickey D (1983) Testing for unit roots in autoregressive moving-average models with unknown order. Biometrika 71:599-607

Soares J, Oliveira AP, Božnar MZ, Mlakar P, Escobedo JF, Machado AJ (2004) Modeling hourly diffuse solar-radiation in the city of São Paulo using a neural-network technique. Appl Energy 79:201-214

Spencer JW (1982) A comparison of methods for estimating hourly diffuse solar radiation from global solar radiation. Sol Energy 29:19-32

Publisher's Note Springer Nature remains neutral with regard to jurisdictional claims in published maps and institutional affiliations. 